\title{
THE INCUBATION PERIOD OF CORONAVIRUS DISEASE 2019 (COVID-19) IN PETALING DISTRICT, MALAYSIA
}

\author{
Ainul Nadziha Mohd Hanafiah ${ }^{1}$, Lee Soo Cheng ${ }^{2}$, Lim Kuang Kuay ${ }^{3}$, Waramlah Ramlan ${ }^{4}$, Diana Raj ${ }^{4}$, \\ Shiehafiel Fieqri Hussin ${ }^{4}$, Ridwan Sanaudi ${ }^{3}$, Roslinda Abu Sapian ${ }^{3}$, Nurul Syarbani Eliana Musa ${ }^{3}$ and \\ Mohamed Paid Yusoff ${ }^{5}$. \\ 1 Institute for Health Systems Research, National Institutes of Health, Ministry of Health, No. 1 Jalan Setia Murni \\ U13/52, Setia Alam, 40170 Shah Alam, Selangor, Malaysia. \\ 2 Petaling District Health Office, Selangor, Ministry of Health, Wisma SAHOCA, No. 1 Jalan SS6/3A, 47301 Petaling \\ Jaya, Selangor, Malaysia. \\ ${ }^{3}$ Institute for Public Health, National Institutes of Health, Ministry of Health, No. 1 Jalan Setia Murni U13/52, Setia \\ Alam, 40170 Shah Alam, Selangor, Malaysia. \\ ${ }^{4}$ Ministry of Health, Level 6, Menara Prisma, Precinct 3, 62675, Putrajaya, Malaysia. \\ ${ }^{5}$ Petaling District Health Office, Selangor, Ministry of Health, Wisma SAHOCA, No. 1 Jalan SS6/3A, 47301 Petaling \\ Jaya, Selangor, Malaysia
}

\section{Corresponding author: Ainul Nadziha Mohd Hanafiah Email: ainulnadziha@gmail.com}

\section{ABSTRACT}

The outbreak of COVID-19 has affected the world with over two million cases globally. Malaysia has witnessed over 5,000 with almost 100 deaths as of 22 April 2020. Knowledge on the incubation period of COVID-19 is scarce due to the novelty of the virus. The aim of this study was to determine the incubation period of COVID-19 infection in the district of Petaling in Malaysia. A cross-sectional study was conducted using secondary data from the Petaling District Health Office. We analysed data of laboratory-confirmed COVID-19 cases notified to the Petaling District Health Office between 3 February 2020 and 13 April 2020. Our study included 219 cases. The median incubation period of COVID-19 among the cases was five days (interquartile range 3.0-8.0). Four main clusters were identified, with significant difference found between two of the clusters. Cluster $A$ has a shorter incubation period 3.5 (3.0-6.8) compared to cluster $B$ as most of the confirmed cases knew the source of infection. COVID-19 incubation period in Petaling, Malaysia fits within the range estimated by the World Health Organization. Based on the upper value of the incubation period interquartile range in our study, the length of quarantine and period of contact tracing should be at least 8 days. This finding is in accordance with our current practice to control the spread of COVID-19.

Keywords: incubation period; COVID-19; coronavirus; Petaling district.

\section{INTRODUCTION}

The world has been deeply affected by the pandemic of the novel coronavirus infection, better known as COVID-19, which started as an epidemic in the city of Wuhan, Hubei province, China in December 2019. COVID-19, which manifests like pneumonia with symptoms such as fever, dry cough, sore throat, myalgia and fatigue $^{1,2}$, is caused by the severe acute respiratory syndrome coronavirus 2 (SARS-CoV-2). Severe fatal complications such as organ failure, septic shock, pulmonary oedema, severe pneumonia, and acute respiratory distress syndrome have also been reported ${ }^{3,4}$.

The World Health Organization declared that the outbreak constituted a Public Health Emergency of International Concern (PHEIC) on 30 January $2020^{5}$. The spread of COVID-19 from the epicenter of Wuhan, China has resulted in 2,471,136 confirmed cases and 169,006 deaths globally as of 22 April 20206. Malaysia has reported 5,532 confirmed cases with 93 deaths $^{7}$, while in Petaling district confirmed cases were 371 with five deaths up until 22 April $2020^{8}$.
The novelty of the coronavirus with unclear clinical spectrum and testing capacity has made it difficult for countries to establish the incubation period of the disease. Knowledge on COVID-19 incubation period is a key epidemiological parameter in establishing and evaluating public health activities such as monitoring, control, and surveillance of infectious diseases, as well as for modelling of epidemics. Incubation period is defined as the time interval between exposure and onset of symptomatic disease ${ }^{9,10}$.

Only a few studies have been conducted to determine the incubation period of COVID-19. Findings were based on epidemiological data from a few countries, but mostly from China. Incubation period have been estimated to range between $3 \cdot 6$ and $6 \cdot 4$ days ${ }^{11-18}$. In Malaysia similar studies have yet to be found. Therefore, the aim of this study was to determine the incubation period of COVID-19 infection in the district of Petaling, Malaysia. Findings from this study will add to the body of knowledge on incubation period of COVID-19 and will assist epidemiologists and the public health fraternity globally in the 
planning of necessary monitoring, control, and prevention measures.

\section{METHODS}

This was a cross-sectional study carried out using data obtained from the Petaling District Health Office, Selangor, Malaysia. Data extracted were those of confirmed cases notified to the Petaling District Health Office between 3 February 2020 and 13 April 2020. Data on symptomatic confirmed cases with known dates of exposure and onset of symptoms were included in this study. Information on socio-demographic characteristics, clinical manifestations, comorbidities, travelling history and source of infection were included in the study.

\section{Study Definitions}

A confirmed COVID-19 case is defined as a person with a laboratory-confirmed positive result ${ }^{19}$. We defined incubation period as the time elapsed between exposure to a confirmed case and the date of onset of symptom(s).

Symptom onset date is defined as the date when the signs or symptoms suggestive of COVID-19 (i.e. cough, shortness of breath and sore throat, with or without fever) first appear ${ }^{19}$, while the date of exposure is defined as the latest date of contact with a confirmed positive case

\section{Statistical analysis}

Statistical analyses were performed using SPSS software version 26 (IBM SPSS, Chicago, IL, USA). No imputation was made for missing data. Categorical variables were summarised as counts and percentages. All continuous variables were statistically tested for normality using the Shapiro-Wilk test before performing any further statistical analysis. Continuous variables such as age and incubation period were expressed as medians and interquartile ranges as both were not normally distributed. Non-parametric test (Kruskal-Wallis test) was performed to compare the differences of incubation periods between the clusters listed in this study. We then performed Dunn's nonparametric comparison for post-hoc testing following a significant Kruskal-Wallis test. The pairwise comparisons using the DunnBonferroni method were produced where the level of significant for adjusted $p$-value is set at 0.05 .

\section{Ethics}

Ethical approval was obtained from the Medical and Research Ethics Committee (MREC), Ministry of Health Malaysia (research registration number: NMRR-20-793-54770). Approval was also obtained from the Petaling Health District Office prior to the study and no findings which could identify any individual will be published.

\section{RESULTS}

A total of 219 cases were included in this study, with $52 \%$ female cases. By age groups, 54\% were aged 40 years old and above; about $70 \%$ cases were Malay, and majority were Malaysians (96\%). We identified four main clusters from the dataset: Cluster A (20.1\%) consists of people from a corporate organisation, Cluster B (19.6\%) consists of people who attended a religious event, Cluster C (33.8\%) are imported cases from abroad, and Cluster D (26.5\%) consists of other categories like individual home clusters or sporadic cases.

In terms of municipality, most were from Petaling Jaya, followed by Shah Alam and Subang Jaya. About $66 \%$ of the transmission cases were local transmission, three quarters of the cases had no travel history (72.1\%) and 63\% did not have comorbidity (Table 1).

Significant differences were observed in the median incubation period of the clusters $(p=0.002)$, whereas, no significant differences were found in age, gender, ethnicity, municipality, transmission, nationality, travelling history, healthcare worker and co-morbidity (Table 1).

Majority of the cases $(70.8 \%)$ had fever, followed by respiratory symptoms (69.4\%). Our study identified the top three co-morbidities of the cases as hypertension (19.2\%), diabetes mellitus $(12.3 \%)$ and heart disease (4.6\%) (Table 1).

The median incubation period of COVID-19 among the cases is 5.0 days (interquartile range 3.0-8.0). The longest median incubation period was found for cluster $B$, which is 8.0 days (interquartile range 4.0-11.0), while the shortest median was for cluster $A$, which is 3.5 days (interquartile range 3.0-6.8). Significant difference was observed between clusters A and B $(p=0.001)$, however, there was no significant difference between the other cluster combinations (Table 2). 
Table 1: The sociodemographic characteristics, clusters, co-morbidity and clinical manifestations of COVID-19 cases

\begin{tabular}{|c|c|c|c|}
\hline \multirow{2}{*}{ Characteristics } & \multicolumn{3}{|c|}{ Incubation Period } \\
\hline & $\mathrm{n}(\%)$ & Median (IQR) & $\mathrm{p}$ \\
\hline \multicolumn{4}{|l|}{ Age } \\
\hline Below 40 & $101(46.1)$ & $6.0(3.5-9.0)$ & \\
\hline 40 and above & $118(53 \cdot 9)$ & $5 \cdot 0(3 \cdot 0-8 \cdot 0)$ & 0.197 \\
\hline \multicolumn{4}{|l|}{ Gender } \\
\hline Male & 105 (47.9) & $6.0(3.5-8.0)$ & \\
\hline Female & $114(52.1)$ & $5.0(3.0-9.0)$ & 0.639 \\
\hline \multicolumn{4}{|l|}{ Ethnicity } \\
\hline Malay & $153(69.9)$ & $5.0(3.0-8.5)$ & \\
\hline Chinese & $41(18.7)$ & $5.0(3.0-7.0)$ & \\
\hline Indian & $14(6.4)$ & $7.5(5.0-9.5)$ & \\
\hline Others & $11(5.0)$ & $7.0(6.0-12.0)$ & 0.111 \\
\hline \multicolumn{4}{|l|}{ Nationality } \\
\hline Malaysian & $210(95.9)$ & $5.0(3.0-8.0)$ & \\
\hline Non-Malaysians & $9(4.1)$ & $7.0(6.0-12.5)$ & 0.062 \\
\hline \multicolumn{4}{|l|}{ Cluster } \\
\hline A & $44(20.1)$ & $3.5(3.0-6.8)$ & \\
\hline B & $43(19.6)$ & $8.0(4.0-11.0)$ & \\
\hline C & $74(33.8)$ & $6 \cdot 0(3.8-8.0)$ & \\
\hline $\mathrm{D}$ & $58(26.5)$ & $5 \cdot 0(3.0-8.0)$ & $0.002^{*}$ \\
\hline \multicolumn{4}{|l|}{ Municipality } \\
\hline Petaling Jaya & $88(40.2)$ & $5.0(3.0-8.0)$ & \\
\hline Shah Alam & $71(32.4)$ & $5.0(3.0-8.0)$ & \\
\hline Subang Jaya & $60(27.4)$ & $6.0(4.0-10.8)$ & $0 \cdot 265$ \\
\hline \multicolumn{4}{|l|}{ Transmission } \\
\hline Local & $145(66.2)$ & $5.0(3.0-9.0)$ & \\
\hline Import & $74(33.8)$ & $6.0(3.8-8.0)$ & 0.788 \\
\hline \multicolumn{4}{|l|}{ Travelling history } \\
\hline Yes & $61(27.9)$ & $5.0(3.0-8.5)$ & \\
\hline No & $158(72.1)$ & $6.0(3.0-8.3)$ & 0.555 \\
\hline \multicolumn{4}{|l|}{ Healthcare worker } \\
\hline Yes & $21(9.6)$ & $6.0(3.0-9.0)$ & \\
\hline No & $198(90.4)$ & $4.0(3.0-8.0)$ & 0.431 \\
\hline \multicolumn{4}{|l|}{ Co-morbidity } \\
\hline Yes & $81(37.0)$ & $6.0(3.0-9.0)$ & \\
\hline No & $138(63.0)$ & $5.0(3.0-8.0)$ & 0.736 \\
\hline \multicolumn{4}{|l|}{ Clinical manifestations } \\
\hline Fever & $155(70.8)$ & & \\
\hline Respiratory symptoms & $152(69.4)$ & & \\
\hline Constitutional symptoms & $39(17.8)$ & & \\
\hline Musculoskeletal symptoms & $14(6.4)$ & & \\
\hline Gastrointestinal symptoms & $10(4.6)$ & & \\
\hline Loss of taste and smell & $7(3.2)$ & & \\
\hline
\end{tabular}


Table 2: The median incubation period of COVID-19 by clusters

\begin{tabular}{llllll}
\hline Cluster & $\mathrm{n}$ & Median & IQR & X2 statistic (df $)^{\mathrm{P}}$ \\
\hline $\mathrm{A}^{\mathrm{a}}$ & 44 & 3.5 & $3.0-6.8$ & & \\
$\mathrm{~B}^{\mathrm{b}}$ & 43 & 8.0 & $4.0-11.0$ & & \\
$\mathrm{C}^{\mathrm{c}}$ & 74 & 6.0 & $3.8-8.0$ & $15.2(3)$ & 0.002 \\
$\mathrm{D}^{\mathrm{d}}$ & 58 & 5.0 & $3.0-8.0$ & & \\
\hline Total & 219 & 5.0 & $3.0-8.0$ & & \\
\hline
\end{tabular}

*Kruskal-Wallis test

Post Hoc tests: ${ }^{a b} p=0.001 ;{ }^{a c} p=0.153 ;{ }^{a d} p=0.478 ;{ }^{b c} p=0.203 ;{ }^{b d} p=0.100 ;{ }^{c d} p=1.00$

\section{DISCUSSION}

From this study, we present data on 219 laboratory-confirmed positive COVID-19 cases in Petaling district. Approximately $70 \%$ of these cases were Malays, which can be explained by the two big clusters, i.e. cluster $A$ and $B$, which are made up of almost all Malays.

The analysis of data yielded a median incubation period of 5 days, which is within the range of incubation period of 0 to 14 days estimated by the WHO, and 2 to 12 days estimated by the European Centre for Disease Prevention and Control $(E C D C)^{20,21}$. Our findings are comparable to the median incubation period values for the severe acute respiratory syndrome (SARS) as well as the Middle East respiratory syndrome (MERS) ${ }^{15,22}$. However, a systematic review reported a shorter median incubation period of 4 days in SARS ${ }^{23}$. Our findings are also consistent with recent estimations of incubation period for COVID-19 in studies in China ${ }^{13,16}$, but is longer than findings from Korea which reported a median incubation period of 3 days, and 5.2 days in a study based on 10 cases in China ${ }^{12,14}$. The variation that we identified in the incubation period distribution between studies could be associated with differences in the ascertainment or reporting of exposure dates and illness onset dates, differences in the source or mode of infection, or environmental differences ${ }^{22}$.

When comparing the incubation periods between clusters in Petaling, we noted that the median incubation periods are significantly different between cluster $\mathrm{B}$ (religious gathering) and cluster A (corporate organisation). Cluster B has the longest median incubation period of 8 days (interquartile range 4.0-11.0) amongst all the clusters in Petaling. This is probably due to the continuous and repeated exposure amongst individuals within the religious cluster as they are a close-knit community. Hence, the exact dates of exposure are often unclear. Apart from Petaling, neighbouring countries like Singapore also reported cases from the same religious group, although no specific incubation period was reported $^{24}$. On the other hand, cluster $A$ has a shorter median incubation period of 3.5 days (interquartile range $3.0-6.8$ ) which concurs with findings of similar studies in Singapore, Korea and China ${ }^{14,18,24}$. Most of the cases in cluster A reported precise exposure dates and the onset of symptoms because majority of the cases were certain of their exposure source and dates by knowing the positive cases they were exposed to. A higher infecting dose or viral load due to direct transmission as well as higher virulence of the strain could possibly lead to a shorter incubation period as reported in SARS and MERS in previous study ${ }^{22,25}$.

In our analysis, both extreme values of incubation period in clusters A and B were 'averaged out' into a midpoint value, thus resulting in the incubation period of 5 days. A similar finding is demonstrated in a previous study comparing incubation periods of SARS between Hong Kong and Toronto ${ }^{25}$.

Clinical manifestations that were commonly reported in our study were fever and respiratory symptoms like cough, sore throat, runny nose and shortness of breath, which concurs with other studies $^{2,18}$. Our study also uncovered reports of neurological symptoms, specifically loss of taste and smell by 7 cases. Although it is not in the COVID-19 symptom list by World Health Organization ${ }^{26}$, a study by Menni et al. ${ }^{27}$ which studied the prevalence of loss of smell and taste among confirmed COVID-19 cases found this feature to be a strong predictor of COVID-19 infection.

Due to the novelty of this virus, several challenges had to be tackled when determining the onset of the disease as some of the confirmed cases presented with atypical clinical manifestations such as gastrointestinal (diarrhoea, abdominal pain), musculoskeletal (myalgia, arthralgia) and other constitutional symptoms (headache, lethargy), which is consistent with findings in other studies, $2,28,29$. Our study also included the 'loss of taste and smell' manifestation when determining the date of clinical onset. A study reported that atypical presentations may have been missed and led to difficulty in diagnosing and controlling the infection ${ }^{12}$. Another challenge we faced was when confirmed positive cases in our study were asymptomatic ( $n=76$; data not shown), which was also found in other studies ${ }^{30-32}$. This 
made it impossible to determine the incubation period for these cases.

The strengths of our study are as follows; firstly, all COVID-19 cases were investigated by trained health personnel and further verified by qualified epidemiologists. Secondly, with the establishment of the clusters from our data, we were able to demonstrate the significant difference in the median incubation period between 2 clusters which dates of exposure were more precise (cluster A) versus less precise dates due to continuous and repeated exposure amongst individuals (cluster B). Thirdly, we included the atypical symptoms in ascertaining the date of onset of symptoms. This enabled us to determine the incubation period, which otherwise would not have been possible if we had followed strictly the list of typical clinical manifestations of COVID-19. The limitation we identified in this study is that data might have been subjected to recall bias, which could affect the accuracy of dates of symptom onset or exposure. This may have resulted in incubation periods which are either too long or too short. Nonetheless, we included all the outliers in the analysis and reported our findings as median.

In conclusion, the median incubation period of COVID-19 in Petaling district is 5 days, which fits within the range estimated by the World Health Organization. Cluster A has a shorter incubation period compared to cluster $B$ as most of the confirmed cases knew the source of infection.

\section{Recommendation}

Currently, the quarantine period for persons exposed to confirmed positive COVID-19 cases is 14 days. Based on the highest median incubation period in our study, medical observation or quarantine period should be of a minimum of 8 days to halt the spread of disease. And we recommend to maintain the current practice of quarantine of 14 days. With the highest value of the incubation period interquartile range being 11 , therefore, our recommendation is justified, and will suffice to curb the spread of disease.

Secondly, in view of the interest of the public health fraternity to improve their monitoring, control, and surveillance activities, further studies should be done to better understand the transmission of COVID-19 in asymptomatic cases.

\section{ACKNOWLEDGEMENTS}

The authors would like to thank the Director General of Health Malaysia in the support of publishing this article. Our special thanks goes to the staff of the Petaling District Health Office.

\section{Conflict of interest}

The authors declared no competing interests. Funding

This study did not require any funds.
Availability of data and materials

The dataset that support the findings of this article belongs to the study. At present, the data are not publicly available but can be obtained from the authors upon reasonable request and with the permission from the Director General of Health, Malaysia.

Authors' contributions

ANMH, LSC and MPY were responsible for the concept, development and supervision of the research. RS, LKK and WR analysed the data; ANMH, DR and SFH constructed the draft manuscript. All authors contributed to the writing of the manuscript, reviewing and approving the final manuscript.

Ethics approval and consent to participate Ethical approval for this study was obtained from the Medical Research Ethic Committee, Malaysia (research registration number: NMRR-20-79354770).

\section{REFERENCES}

1. Shim E, Tariq A, Choi W, et al. Transmission potential and severity of COVID-19 in South Korea. Int J Infect Dis. 2020;93:33-344.

2. Huang C, Wang $Y$, Li X, et al. Clinical features of patients infected with 2019 novel coronavirus in Wuhan, China. Lancet. 2020;395(10223):497-506.

3. Sohrabi C, Alsafi Z, O’Neill N, et al. World Health Organization declares global emergency: A review of the 2019 novel coronavirus (COVID-19). International Journal of Surgery. 2020;76:71-76.

4. Zhou M, Zhang X, Qu J. Coronavirus disease 2019 (COVID-19): a clinical update. Front Med. 2020;14(2):126-135.

5. World Health Organization. Statement on the meeting of the International Health Regulations (2005) Emergency Committee regarding the outbreak of novel coronavirus (2019-nCoV). WHO Newsletter. 2020. Available from: https: / /www. who.int/newsroom/detail/30-01-2020-statement-onthe-second-meeting-of-the-internationalhealth-regulations-(2005)-emergencycommittee-regarding-the-outbreak-ofnovel-coronavirus-(2019-ncov) (Accessed 23 April 2020).

6. World Health Organization. Coronavirus disease 2019 (COVID-19) Situation Report - 93. World Health Organization. 2020. Available from: https://www.who.int/emergencies/disea 
ses/novel-coronavirus-2019 (Accessed 23 April 2020).

7. Ministry of Health Malaysia. Kenyataan Akhbar KKM: Situasi Semasa Jangkitan Penyakit Coronavirus 2019 (COVID-19) di Malaysia 22 April 2020. Available from: https: / /kpkesihatan.com/2020/04/22/ke nyataan-akhbar-kpk-22-april-2020situasi-semasa-jangkitan-penyakitcoronavirus-2019-covid-19-di-malaysia. (Accessed 23 April 2020).

8. Petaling District Health Office. Laporan Harian Wabak COVID-19, Daerah Petaling, 22 April 2020. 2020. (unpublished).

9. Brookmeyer R. Incubation Period of Infectious Diseases. Wiley StatsRef Stat Ref Online. 2015;1-8.

10. Nishiura H. Early efforts in modeling the incubation period of infectious diseases with an acute course of illness. Emerg Themes Epidemiol. 2007;4:1-12.

11. Linton NM, Kobayashi T, Yang $Y$, et al. Incubation Period and Other Epidemiological Characteristics of 2019 Novel Coronavirus Infections with Right Truncation: A Statistical Analysis of Publicly Available Case Data. J Clin Med. 2020;9(2):538.

12. Li Q, Guan X, Wu P, et al. Early Transmission Dynamics in Wuhan, China, of Novel Coronavirus-Infected Pneumonia. N Engl J Med. 2020;382(13):1199-207.

13. Backer JA, Klinkenberg D, Wallinga J. Incubation period of 2019 novel coronavirus (2019- nCoV) infections among travellers from Wuhan, China, 2028 January 2020. Eurosurveillance. 2020;25(5):1-6.

14. Ki M. Epidemiologic characteristics of early cases with 2019 novel coronavirus (2019-nCoV) disease in Korea. Epidemiol Health. 2020;42:e2020007.

15. Jiang X, Rayner S, Luo MH. Does SARSCoV-2 has a longer incubation period than SARS and MERS? Journal of Medical Virology. 2020;92(5):476-8.

16. Lauer SA, Grantz KH, Bi Q, Jones FK, Zheng Q, Meredith HR, et al. The Incubation Period of Coronavirus Disease 2019 (COVID-19) From Publicly Reported Confirmed Cases: Estimation and Application. Ann Intern Med. 2020;172(9):577-582.
17. Zhang J, Litvinova $M$, Wang $W$, et al. Evolving epidemiology and transmission dynamics of coronavirus disease 2019 outside Hubei province, China: a descriptive and modelling study. Lancet Infect Dis. 2020;3099(20):1-10.

18. Guan W, Ni Z, Hu Y, et al. Clinical Characteristics of Coronavirus Disease 2019 in China. $N$ Engl J Med. 2020;382:1708-1720.

19. Ministry of Health Malaysia. Guidelines on COVID-19 Management in Malaysia No. 5/2020. 2020.

20. World Health Organization. Coronavirus disease 2019 Pandemic. World Health Organization. 2020. Available from: https://www.who.int/emergencies/disea ses/novel-coronavirus-2019. (Accessed 24 April 2020).

21. European Centre for Disease Prevention and Control. Q\& A on COVID-19. European Centre for Disease Prevention and Control. 2020. (Accessed 24 April 2020).

22. Virlogeux V, Fang VJ, Park $M$, et al. Comparison of incubation period distribution of human infections with MERS-CoV in South Korea and Saudi Arabia. Sci Rep. 2016;6(1):35839.

23. Lessler J, Reich NG, Brookmeyer R, et al. Incubation periods of acute respiratory viral infections: a systematic review. Lancet Infect Dis. 2009;9:291-300.

24. Pung $\mathrm{R}$, Chiew $\mathrm{CJ}$, Young BE, et al. Investigation of three clusters of COVID19 in Singapore: implications for surveillance and response measures. Lancet. 2020;395(10229):1039-1046.

25. Cowling BJ, Muller MP, Wong IOL, et al. Alternative Methods of Estimating an Incubation Distribution Examples from Severe Acute Respiratory Syndrome. Epidemiology. 2007;18(2):253-259.

26. World Health Organization. Q\&A on coronaviruses (COVID-19). World Health Organization. 2020. https: //www. who.int/emergencies/disea ses/novel-coronavirus-2019/questionand-answers-hub/q-a-detail/q-acoronaviruses. (Accessed 29 April 2020).

27. Menni C, Valdes A, Freydin MB, et al. Loss of smell and taste in combination with other symptoms is a strong predictor of COVID-19 infection. medRxiv. 2020 
28. Pan $L$, Mu M, Yang $P$, et al. Clinical Characteristics of COVID-19 Patients with Digestive Symptoms in Hubei, China. Am J Gastroenterol. 2020;115:766-773.

29. Siegel A, Chang PJ, Jarou ZJ, et al. Lung Base Findings of Coronavirus Disease (COVID-19) on Abdominal CT in Patients With Predominant Gastrointestinal Symptoms. 2020;215:1-3.

30. Hu Z, Song $\mathrm{C}, \mathrm{Xu} \mathrm{C}$, et al. Clinical Characteristics of 24 Asymptomatic Infections with COVID-19 Screened Among
Close Contacts in Nanjing, China. Sci China Life Sci. 2020;63(5):706-711.

31. Bai Y, Yao L, Wei T, et al. Presumed asymptomatic carrier transmission of COVID-19. J Am Med Assoc. 2020;323(14):1406-1407.

32. Arons $M M$, Hatfield KM, Reddy SC, et al. Presymptomatic SARS-CoV-2 Infections and Transmission in a Skilled Nursing Facility. N Engl J Med. 2020;382(22):2081-2090. 